une revue Gallia

Rhône-Alpes | 2007

\title{
Vienne
}

13 - 15, rue Pégeron

\section{Catherine Coquidé}

\section{(2) OpenEdition}

12 Journals

Édition électronique

URL : http://journals.openedition.org/adlfi/6862

ISSN : 2114-0502

Éditeur

Ministère de la culture

Référence électronique

Catherine Coquidé, "Vienne », ADLFI. Archéologie de la France - Informations [En ligne], Rhône-Alpes, mis en ligne le 01 mars 2007, consulté le 22 avril 2019. URL : http://journals.openedition.org/ adlfi/6862

Ce document a été généré automatiquement le 22 avril 2019

(c) Ministère de la Culture et de la Communication, CNRS 


\title{
Vienne
}

\author{
13 - 15, rue Pégeron
}

\section{Catherine Coquidé}

\section{Identifiant de l'opération archéologique : 229640}

Date de l'opération : 2007 (EX)

1 Un diagnostic a permis l'évaluation archéologique d'une parcelle de $5000 \mathrm{~m}^{2}$ située à proximité du Rhône sur la rive gauche. L'absence de côte-plancher a permis l'exploration du substrat sur plus de $4 \mathrm{~m}$ de profondeur et la pose de jalons chronologiques. Cette opération qui représente la première opportunité d'étude du sous-sol et de l'environnement sur ce secteur se situe aux limites nord de la ville antique.

2 Les indications géologiques de la carte du BRGM signalent une formation holocène, une mise en place relativement récente du substrat dont la base archéologiquement stérile restait à définir. Le plancher des décapages s'est finalement fixé sur un cailloutis dont le toit irrégulier présente un pendage général nord-est - sud-ouest. La nappe phréatique y apparaît au point le plus bas dans l'angle sud-ouest de la parcelle (sondage 1). Rien ne permet de discriminer l'origine de ce dépôt (terrasses wûrmiennes et cône de déjection de la Sévenne côté est ou variation du cours du fleuve côté ouest). Occasionnellement colmatée d'argiles plus ou moins bariolées ou plus fréquemment de lits de sables grossiers, cette base hydromorphe sous-tend d'épais limons argileux jaunes qui atténuent peu à peu la dénivelée. De rares tessons non tournés et quelques inclusions de terre cuite s'insèrent dans les deux tiers supérieurs de ces formations qui sont sans doute à associer à la plaine d'inondation du fleuve. Leur sommet sous-tend une occupation antique scellée par deux niveaux de labours successifs dont le plus récent, de teinte plus foncée, livre quelques tessons de céramiques contemporaines. Côté ouest, cette épaisse terre végétale tend à disparaitre au profit de dépôts plus ou moins pollués issus de l'histoire industrielle des lieux. À noter que les premiers ruissellements issus du démantèlement de formations caillouteuses en amont interviennent à partir du sommet des limons jaunes et se 
succéderont jusqu'à l'interface des deux labours, la mise en place de la voie ferrée barrant le versant étant peut-être à l'origine de ce récent assainissement.

3 Le site antique a été repéré sur le tiers nord de l'emprise (sondages 9 à 11). Il intervient au sommet de limons jaunes déjà plus ou moins anthropisés et se développe sur $0,40 \mathrm{~m}$ à $0,60 \mathrm{~m}$ de hauteur. Le toit des vestiges apparaît à la base du labour entre $1,80 \mathrm{~m}$ et 2,10 $\mathrm{m}$ de profondeur, soit entre $151,25 \mathrm{~m}$ et 151,55 m NGF. Une auréole de mobilier hors contexte est enregistrée sur les sondages voisins; côté ouest (sondage 8), un fin niveau antique colluvié est colmaté par ces mêmes dépôts limoneux témoignant d'un environnement encore en formation. Les ruissellements qui apparaissent à partir de la base de cette occupation restent perceptibles jusque dans les niveaux de terres végétales et confirment l'instabilité du milieu et ce jusqu'à une période récente.

On distingue plusieurs phases d'occupation alternant avec la pose de remblais. Les comblements des vestiges les plus anciens, quelques fosses et un drain, sont relativement stériles. Un ruissellement bouche ces ouvertures puis un remblai, cette fois très anthropisé, exhausse la surface. On enregistre alors la mise en place de plusieurs foyers ainsi que de nombreuses traces de rubéfaction éparses, de nombreux fragments d'amphores et de dolia. Un fond d'amphore coffré d'une argile bleue est observé in situ ; un large foyer circulaire reçoit une épaisse semelle de mortier pouvant éventuellement accueillir et stabiliser un récipient de stockage. Un ou deux solins interfèrent lors de cette phase alors qu'un mur de clôture (?) nord-sud intervient sur les abords ouest. Après la pose d'un nouveau remblai, un niveau de circulation formé de divers éléments jointifs ( tegulae, dolia, amphores) ainsi qu'une nouvelle passée graveleuse colmatent l'ensemble. Le mobilier issu des niveaux supérieurs de la stratigraphie est précoce (Auguste); la céramique fine ne représente que $8 \%$ du total. Aucun élément d'activité particulière n'est observé in situ. On remarque néanmoins un élément de mouture en tegula taillée (sondage 8 , hors contexte antique) et une cale utilisée lors d'activités potières.

5 Le lien entre le site mis au jour et les mosaïques détruites une cinquantaine de mètres au nord-ouest n'est pas évident. L'occupation de cette partie de la rive gauche pourrait s'apparenter à celle d'un suburbium où coexiste un certain nombre d'activité, de la villa ou la domus suburbaine jusqu'à la production spécialisée de denrées (Bedon, 1998). Les vestiges et le mobilier mis au jour lors du diagnostic semblent illustrer une activité tournée vers le stockage et la cuisson. Ils peuvent s'intégrer à la pars rustica d'une exploitation autonome ou être au cour d'un commerce en direction de la capitale de cité des Allobroges.

6 Aucune autre occupation n'est enregistrée jusqu'à l'époque contemporaine. L'étude environnementale mentionne que la parcelle accueille d'abord une activité de filature avant le rachat des locaux au milieu du $\mathrm{XX}^{\mathrm{e}} \mathrm{s}$. À partir de cette date, on signale le démantèlement des machines et la mise en place d'un lieu de stockage pour les tissus puis l'installation d'un garage.

7 Les niveaux contemporains enregistrés lors du diagnostic permettent de préciser l'utilisation de ces espaces. Le mobilier issu du sommet de la terre végétale ou des premiers niveaux pollués indique le XIX ${ }^{e} \mathrm{~s}$. avec une majorité de production de la seconde moitié du siècle. La coupe synthétique montre un relief encore ondulé que les premiers remblais de démolition gomment définitivement.

8 Le premier bâti s'intègre déjà à un environnement industriel (quasi-absence de la vaisselle commune et présence de scories de fer). Il n'est observé que sur la moitié ouest 
de la parcelle le long de la rue Pégeron. Les espaces arrière masquent alors une succession d'épaisses strates industrielles de type scories de verre régulièrement intercalées de remblais limonograveleux. Celles-ci sont probablement déjà en place au début du XX $\mathrm{X}$. comme en témoigne la hauteur des graffitis relevés sur les murs est (le long de la voie ferrée) et sud-est. Leur présence signale un espace arrière en aire ouverte alors que les dates, commentaires et personnages illustrent une séquence chronologique située entre 1913 et 1946, celle des années 1920 faisant référence à une fréquentation militaire. Le bâti détruit préalablement à notre intervention concerne quant à lui les trois quarts arrière de la parcelle et ménage une cour côté voirie. Sa construction oblitère totalement l'ancienne occupation du sol.

9 COQUIDÉ Catherine

INDEX

peuple Allobroge

operation expertise (EX)

Index chronologique : Holocène, Temps Modernes, ép contemporaine, Haut-Empire, Empire romain

Index géographique : Rhône-Alpes, Isère, Vienne

\section{AUTEURS}

CATHERINE COQUIDÉ 